\title{
Common Lumbar and Sacral Interventional Pain Procedures
}

\author{
Milan Mehta ${ }^{1}$, Bharat $\mathrm{J} \mathrm{Shah}^{1}$, Palak Chudasama ${ }^{1}$
}

\section{Abstract}

Interventional pain specialists perform all pain procedures under live fluoroscopic guidance and with use of a contrast agent to deliver cortisone as close to the disc herniation or nerve root impingement, as determined by MRI or at the pain generators. Image guided technique reduces morbidity. Dye spread in AP, lateral and oblique view is to be documented in real time to reduce catastrophic complications. It outlines area surrounding exiting and traversing nerve roots along with epidural space and also delineates perimeter of joint.

All these procedures help in identifying pain generators by means of diagnostic techniques which helps in conservative management as well as it's very useful before radio-frequency ablative procedures and before surgery.

Keywords: Lumbar and sacral pain; Non operative treatment; Intervention pain speciality.

\section{Introduction}

Epidural nerve block consists of the administration of small volumes of target-specific local anesthetics, corticosteroids, and other agents into the epidural space to interrupt the pain cycle and reduce inflammation of either axial or radicular pain [1].

Interventional pain specialists perform under fluoroscopic guidance and with use of a contrast agent in order to deliver cortisone as close to the disc herniation or nerve root impingement, as determined by MRI. Image guided technique reduces morbidity.

It is useful for management of acute, subacute, or chronic axial spine or radicular pain that is refractory to more conservative care such as rest, analgesics, and physical therapy [2].

There are interlaminar, transforaminal and caudal approaches to access epidural space. It reduces leg pain, improve leg raising, and improve lumbar flexion at 2 weeks after injection in many patients. Long term benefits are not proven. Procedural morbidity also varies with each approach. With interlaminar injections, there is a risk of intrathecal injection and subsequent arachnoiditis as well as postprocedural headache. Patients receiving transforaminal injections frequently report transient leg pain, and there is a risk of spinal cord infarction

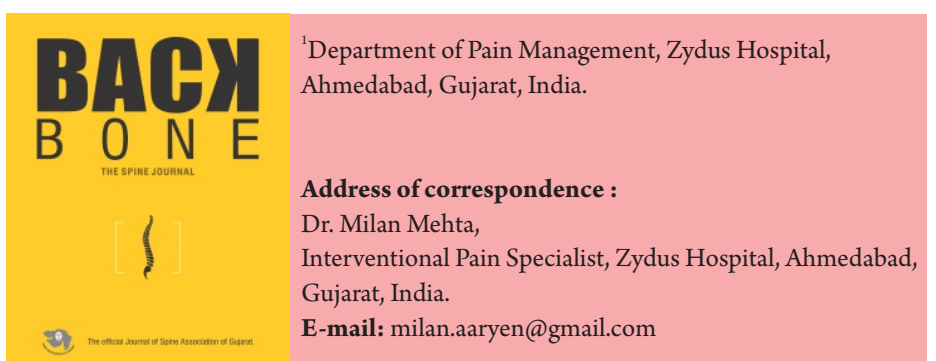

[3].

A definite trend toward nonsurgical management of lumbosacral disc herniation with radicular symptoms has been noted due to following reasons [4]:

1) Resorption of lumbar herniated disc fragments can occur.

2) Some lumbar disc disorders are asymptomatic, including herniations.

3) There is an inflammatory component to low back and radicular pain in the presence of disc herniation; an inflammation reaction may be necessary to precipitate symptoms

The following issues should be kept in mind during an epidural block [5]:

1) If the patient experiences any significant pain or there is a sudden increase in resistance during injection, the needle position may be incorrect; the injection should be stopped immediately.

2) If cerebrospinal fluid is aspirated, the epidural block should be postponed.

3) If aspiration of blood occurs, needle placement should be adjusted.

\section{Interlaminar Epidural [6] [7]}

Procedure in which needle is passed through interlaminar space and reaches to the dorsal epidural space. Main application is to deposit drugs either directly or via catheter for continuous infusion and to guide electrodes for spinal cord stimulators.

Now a days fluoroscopy guided injections are recommended to avoid pitfalls of blind technique, however it retains some features of blind technique as well. 
Blunt tip tuohy needle is advisable as it clearly identifies resistance of ligamentum flavum, also pushes dural sac away and loss of resistance is much more evident.

This procedure is contraindicated in patients with localised infection or coagulation abnormalities

\section{Technique}

Patient should lie in prone with pillow below iliac crest/ abdomen to reduce lumbar lordosis.

All sterile technique is to be applied. AP view of the targeted segment should be taken first. Cranio-caudal tilt should be adjusted in such a way that targeted space is widely open. Interlaminar space is radiolucent. Needle should be directed onto the lamina just below the targeted space, this will give an idea about depth of epidural space. Now needle should be redirected towards ligamentum flavum and saline filled loss of resistance (LOR) syringe should be connected to check loss of resistance. Depth of needle should be checked frequently in lateral and contralateral oblique views. In lateral views tip of needle should lie within shadow of inferior articular process and posterior to vertebral canal. Once successful loss of resistance is achieved, radiopaque dye should be injected and spread pattern should be observed in live fluoroscopy in AP and lateral views. Contrast should form smooth but irregular pattern that appears lobulated due to epidural fat. More contrast delineate root sleeve. It is must to identify vascular uptake, subdural or intrathecal spread to prevent major complications.

There is no consistency in literature as to the type of steroid and volume or dose to be injected. 2 to $5 \mathrm{ml}$ of volume comprise of local anaestheic along with triamcinolone or depomedrol or dexamethasone. Depot preparations are contraindicated for cervical transforaminal placements. Many practitioners use only non particulate steroids. The rational to use steroid is to treat lumbar radicular pain. Evidence of efficacy is lacking. Steroid injections are no more effective than injection of local anesthetic alone.

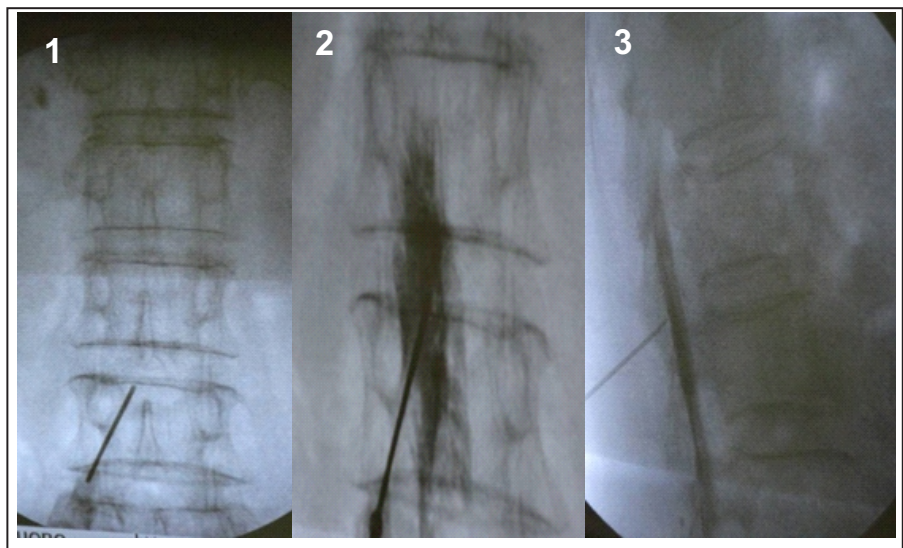

Figure 1: (1) Access to Lumbar Epidural Space (2) Dye spread in APview (3) Dye spread in lateral view

\section{Caudal Epidural $[6][7]$}

The caudal epidural injection is very well known technique for epidural steroid injection.

There are rudimentary spinous processes from the first three or four sacral segments in the midline. The laminae of the fifth sacral vertebra fail to unite posteriorly to form a sacral hiatus. which is U- or V-shaped. The sacral hiatus is covered by the sacrococcygeal membrane. Just beneath the sacrococcygeal membrane lies the caudal border of the epidural space.

The termination of the thecal sac varies depending on age, and varies between the lower border of the $\mathrm{S} 1$ foramen in adults and the $\mathrm{S} 3$ foramen in children. This is important while placement of the epidural needle tip and avoiding dural puncture. However this is best area to target multiple nerve roots in the anterior epidural space which is the commonest area for most pathology. Anterior epidural spread can be achieved by specialized catheter or with high volume and can be seen by radio-opaque dye spread.

\section{Technique}

Procedure is to be done in prone position under local anaesthesia under fluoroscopy.

Insert a 22 gauge, $3 \frac{1 / 2}{2}$ inch needle toward the inferior lateral angle of the sacral cornua, approximately at a $45^{\circ}$ angle to the skin, through the sacrococcygeal membrane into the caudal epidural space. A loss of resistance will occur while the needle enters the caudal space. Advance the needle $2 \mathrm{~cm}$ more cephalad into the caudal epidural space with the needle between the dorsal and ventral plates of the sacrum. There has to be negative aspiration for blood and CSF. Radio-opaque dye should delineate Christmas tree pattern without vascular uptake. Local along with steroid can be injected for

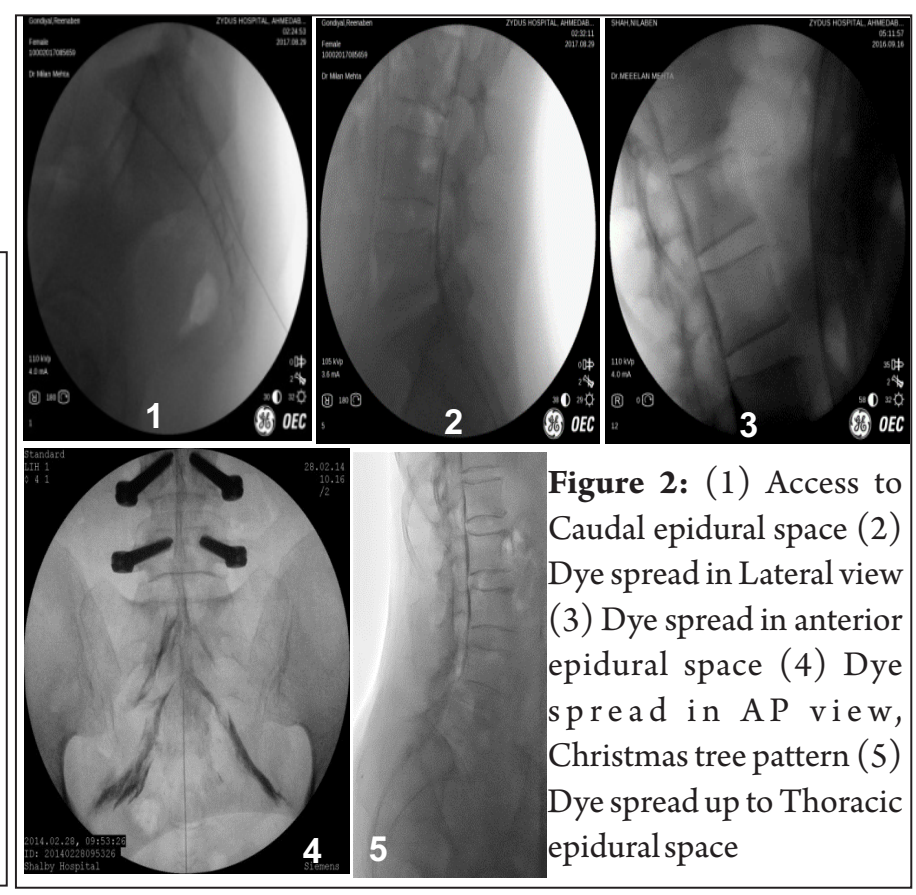

34 | Back Bone: The Spine Journal | Volume 1 | Issue 1 | April-September 2021 | Page 33-42 
symptomatic relief of radicular symptoms. Intraocular pressure can be increased and haemorrhage has been reported. Caudal epidural adhesinolysis is very effective procedure to treat postoperative fibrosis. Higher spread up to thoracic level can be seen many a times.

\section{Transforaminal Epidural Block (tfeb) [6] [7]}

Spread of an injectate through the epidural space as well as along the spinal nerve.

It is used therapeutically because the injectate spreads into the anterior epidural space, which is the perceived target site of the disease. Local anesthetics with or without steroid can be used. TFEB injections can control inflammation and can stabilize sensitized nociceptive neural activity due to the many different pain sources originating in the spine.

TFEB requires a lower volume $(1-3 \mathrm{~mL})$ of injectate than conventional epidural block

If the initial response to TFEB is favourable but short-lived, a series of injections (3-6 times per year) or pulsed radiofrequency lesioning of the corresponding dorsal root ganglion (DRG) is recommended.

\section{Selective Nerve Root Block (SNRB) [6] [7]}

Spread of injectate not into the epidural space but along the spinal nerve. It is used for diagnostic purposes.

SNRB may be particularly useful in identifying the symptomatic level in patients with multilevel pathology.

\section{Indications}

\section{Indications for TFEB are as follows}

- Nonsurgical treatment of atraumatic spondylotic or traumatic spondylotic radicular pain

- Radicular pain of postoperative scarring

- Radicular pain when a surgically correctable lesion cannot be found

- Radicular pain when urgent surgery either is not indicated or is contraindicated by medical conditions

- Spinal stenosis-especially in symptomatic foraminal stenosis

\section{Indications for SNRB are as follows}

- To identify nerve root(s) responsible for pain when clinical or radiographic findings are equivocal

- To confirm the pain generator in failed back surgery syndrome with extremity pain

-For planning of surgical treatment

\section{Contraindications}

Contraindications to both TFEB and SNRB are as follows

- Patient refusal

- Coagulopathy
- Allergy to injectates

- Therapeutic anticoagulation (aspirin and antiplatelet agent therapy should be stopped 7 days, and warfarin therapy 5 days, before TFEB or SNRB)

- Skin infection at the injection site

- Raised intracranial pressure

- Pregnancy

\section{Technique}

Patient lies prone on fluoroscopy table. First scan in PA view. Inferior endplate should be squared off of upper vertebra of target segment. Target point is $6^{\circ}$ clock of the pedicle.

Safe triangle is to be identified. If the target point is seen on PA view and which is not covered by superior articular process, transverse process or lamina, direct approach to the needle is possible for access. Tilt the c-arm obliquely until entry point is not covered or shadowed by any bony structure. Puncture point on skin is selected. After local anaesthetic infiltration, 23G lumber puncture needle is advanced towards target point; advancement of needle is frequently checked by c-arm views. Needle should be advanced till it hits the bone at target point.

Touching the nerve and eliciting paresthesia should not be attempted, however if needle touches, withdrawal of few millimetre is necessary. If pain doesn't settle, procedure should be abandoned.

This technique is for targeting nerve root in safe triangle by standard subpedicular technique, however if we encounter nerve root, we will have to reposition our needle by retro neural approach. Once the needle reaches the target point, tip should be checked in AP and Lateral views. 0.2 to $0.3 \mathrm{ml}$ non-ionic contrast should be injected in live fluoroscopy as large volume might compresses the nerve. We will have to rule out vascular uptake, if it is found, procedure should be abandoned as adjustment of needle doesn't give guarantee. DSA mode is very useful to rule out vascular uptake. Ideal spread would be along the targeted nerve, delineate both exiting and traversing nerve roots and root sleeve. If too much peripheral spread is found, redirect the needle medically. Contrast should reach culprit disc which is pressing the nerve to reduce the inflammation. Bolus of any injectate should reach disc-nerve interface. Contrast must be injected in live fluoroscopy both in AP and Lateral views.

Lot of studies suggest that major reinforcing arteries to spinal cord arises at thoracic level. In approximately $95 \%$ of the times It is found between $\mathrm{T} 8$ to $\mathrm{L} 2$ segment but it can be anywhere between T5 to L5 segment. In some studies it is also found accompanying sacral nerves.

To access the foramen, Subpedicular approach is standard one but with far lateral herniations and with foraminal stenosis, nerve root is displaced in upward direction, hence safe triangle will no more be safe. Other approaches to access the foramen 


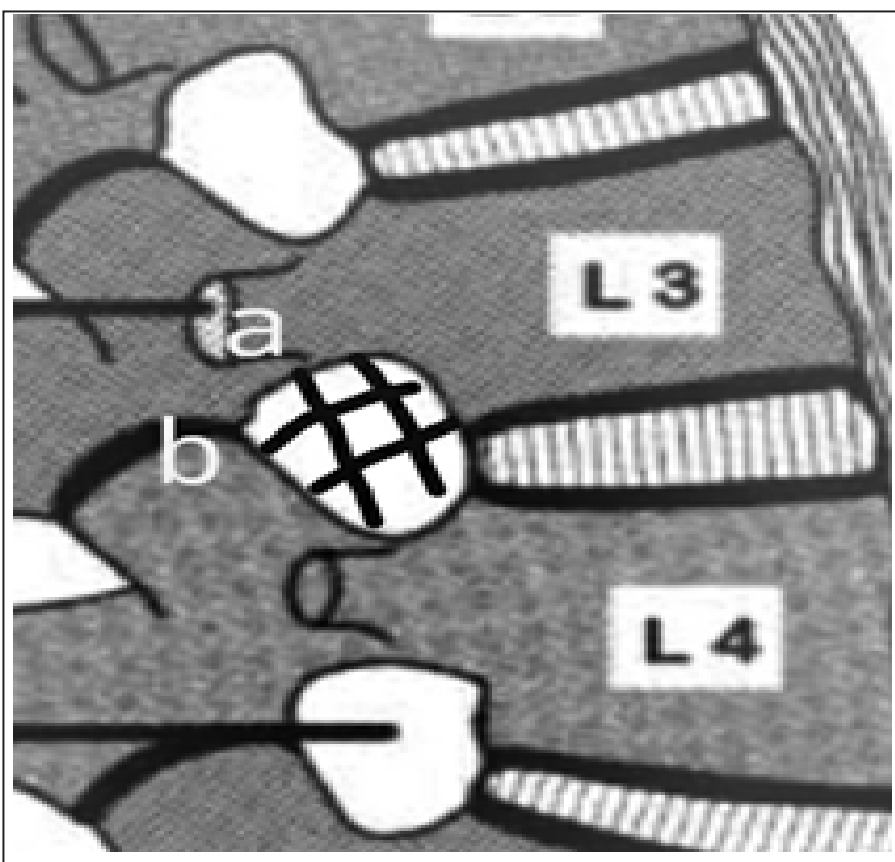

Figure 3:

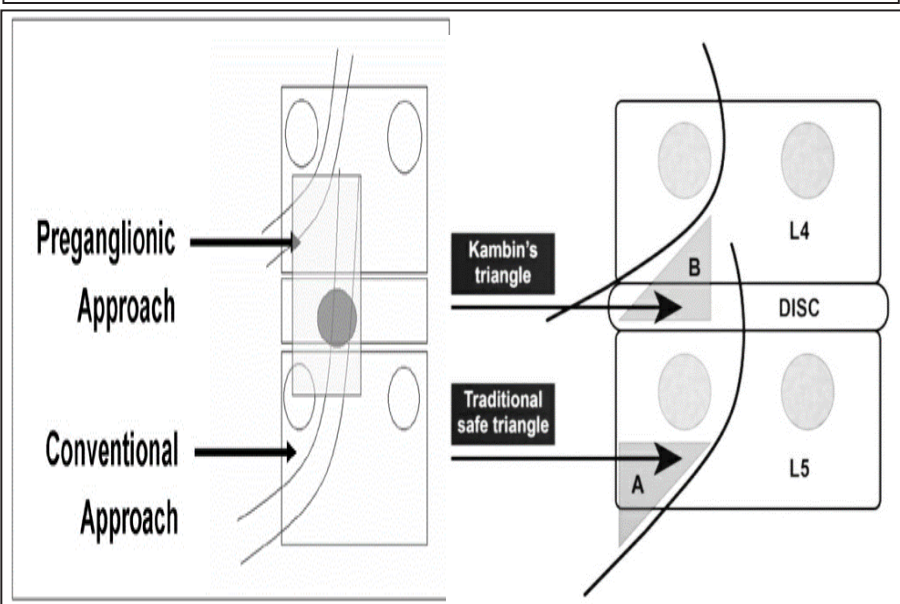

Figure 4: Three transforaminal approaches. (A) Anteroposterior view (B) Lateral view. B: vertebral body, D: intervertebral disc, $\mathrm{N}$ : spinal nerve, $\mathrm{P}$ : pedicle

are Superaneural, Retroneural and infraneural for that planning should be done based on MRI to prevent direct needle injury.

\section{Retroneural Approach}

Target point lies at the intersection of two lines $a$ and $b$ where (a) is longitudinal line between middle and posterior third of foramen and (b) is transverse line between upper and middle third of foramen. In AP views, it lies at opposite the midpoint of lateral edge oflamina.

\section{Supraneural Approach}

It is hybride of Subpedicular and Retroneural approach. In AP

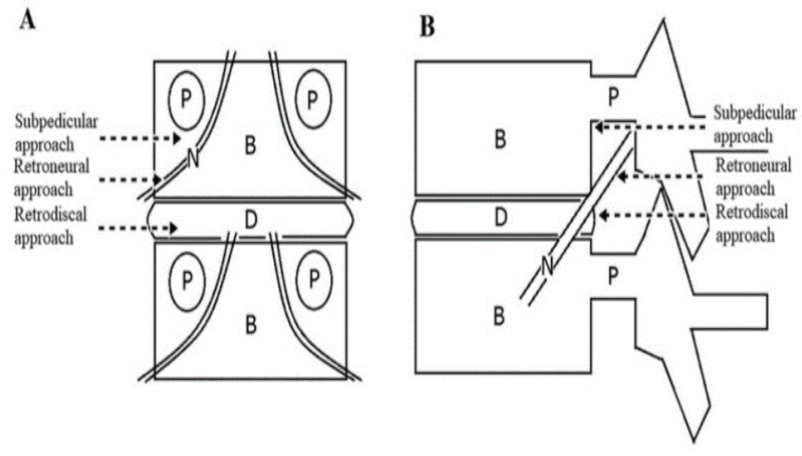

Fig. 1. Three transforaminal approaches. (A) Anteroposterior riew (B) Lateral vieu.

B: vertebral body, D: intervertebral disc, N: spinal nerve, P: pedide

\section{Figure 5:}

view, it is at subpedicular $6^{\circ}$ clock position where as in lateral view, it is much superficial.

\section{Infraneural Technique}

Also known as retrodiscal approach. Needle is aimed towards disc. Final target is at lower half of intervertebral foramen between disc in front and facet joint behind. Chances of Intradiscal spread is very high.

\section{Complications}

1.Infection: epidural abscess, meningitis

2. Bleeding: epidural hematoma

3. Allergic reaction to injectates

4. Dural puncture (0.5\%-2.5\% of cases), dural puncture headache

5. Intravascular injection

6. Post-injection exacerbation of pain ( $1 \%$ of cases)

7. Arachnoiditis with certain preparations of steroids

8. Suppression of plasma cortisol levels for up to 2 weeks

9. Increase in blood glucose level from steroid injection

10. Neurologic complications due to direct nerve trauma

11. Paraplegia is most dangerous complication as size of particulate steroid particles are larger compare to RBC which act as vascular emboli, eventually block the anterior spinal artery via feeder vessel.

Selective Nerve Root Block (SNRB) is performed by means of the same technique as TFEB, except for the final position of the needle tip. The target points for L1 to L5 SNRB are located more laterally than those for TFEB, to avoid epidural spread of the injectate. The target points for L1 to L5 SNRB also differ according to the vertebral level involved when the needle is close to the nerve root; the needle is advanced slowly until it is adequately placed at the target or until the patient experiences paresthesia. Provocation of paresthesia is not mandatory for an SNRB. 


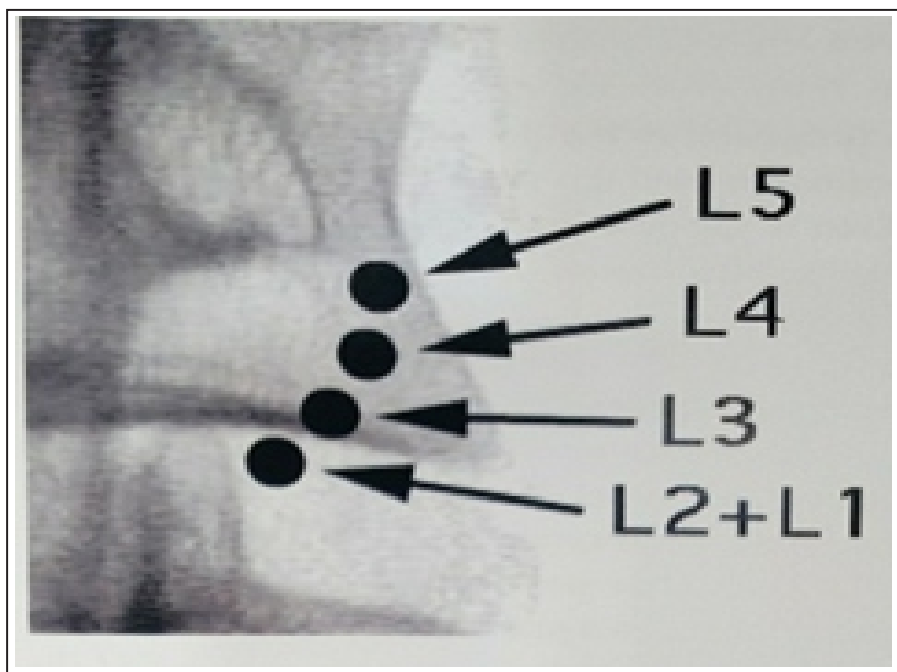

Figure 6: Schematic drawing of the target points for diagnostic nerve blocks at the various levels.(Oblique projection)

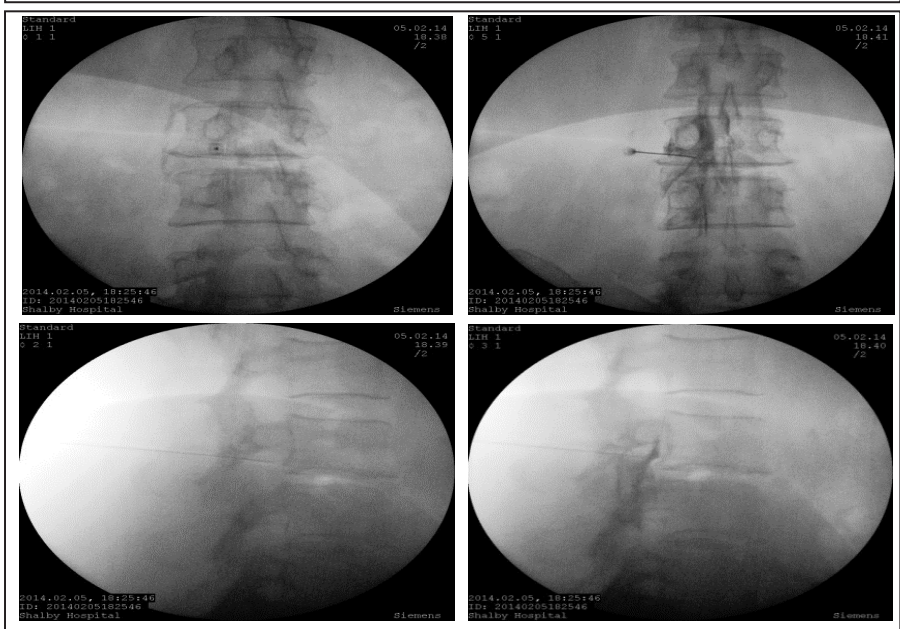

Figure 7: (1) Sub pedicular 6o'clock entry point (2) Dye spread is seen which delineate Epidural space along with exiting and traversing nerve root (3) End point of needle in lateral view (4) Dye spread in lateral view

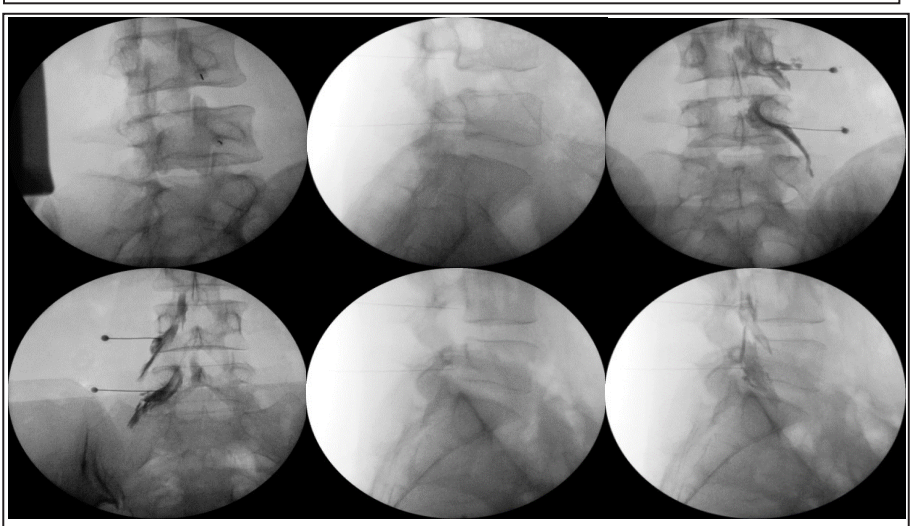

Figure 8: Transforaminal epidural spread

(1) Needle tip in oblique view (2) Needle inside the foramen at L4-5 and L5-S1 level (3) Dye spread shows right side L4 and L5 nerve root (4) Dye spread shows left side L4 and L5 nerve root (5) Lateral view without dye spread (6) Lateral view with dye spread

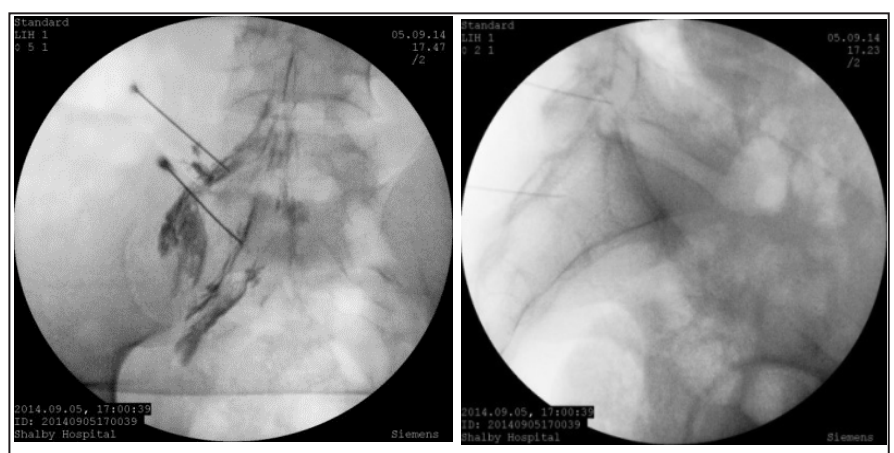

Figure 9: Pulsed Radiofrequency of Left L5 Dorsal Root Ganglion and $\mathrm{S} 1$ nerve root

1 APview 2 Lateral view

When the patient experiences paresthesia, $0.5 \mathrm{ml}$ of contrast dye is injected under fluoroscopic guidance using an AP image. This injection confirms that the needle tip is appropriately near the nerve root and ensures that the injectate is not passing epidurally or intravascularly after proper localization of the needle tip is confirmed; $0.5 \mathrm{ml}$ of local anesthetic is injected through the needle.

\section{Subpedicular Approach For S1 [6] [7]}

The target point for S1 transforaminal injection lies on the caudal border of $S 1$ pedicle.

Posterior sacral foramina are obliquely projected and it is short channel rather than a simple foramen.

First scout image is taken, followed by end plate square off and finally oblique tilt.

Transverse sacral image is helpful in deciding obliquity of posterior sacral foramen.

That much lateral tilt is required for an alignment. Posterior foramen is smaller and circular whereas anterior foramen is larger and ovoid. Medial and inferior margin

of S1 pedicle is seen as thin dark line, which can be easily identified. S1 nerve root sleeve run medial and parallel to this line. Rest of the technique remains the same.

Before the diagnostic block, patient should having pain to anticipate meaningful result following diagnostic block. Positive response can be evident in 10 minutes. It is considered ineffective if fail to relieve symptoms after 30 minutes.

Patient should be able to perform activities which was painful prior to block. One immediate assessment and another at 30 mins interval should be carried out. If relief is observed but return of pain comes after $30 \mathrm{~min}$. will be considered as negative block. Complete relief of symptoms and which lasts for the duration of LA should be considered as positive response. If the patient' pain is mediated by nerve or nerves which are anaesthetized, there should be complete relief. If pain is mediated by more nerves patient may experience partial relief or if the pain persists means pain is not mediated by the nerve which is blocked. 
It is preferable to diagnose multilevel pain as staged procedure. For bilateral diagnostic block careful examination is required. Transforaminal injection of steroids is a procedure to treat radicular pain.

There are strong evidences which tells that inflammatory process plays a key role in producing radicular symptoms due to disc herniation. Injection of steroids is logical and attractive form of intervention. Transforaminal administration delivers the maximum concentration of drug close to or exactly at the site of pathology. Particulate vs nonparticulate (methyl prednisolone $40 \mathrm{mg}$, triamcinolone $40 \mathrm{mg}$, dexamethasone 6 to $8 \mathrm{mg}$ and betamethasone $3 \mathrm{mg}$ ). Manufacturers have already warned against epidural use of particulate steroids, despite of that it is frequently used across the world. Unrecognised or accidental injection into thecal sac leads to arachnoiditis. Unrecognised intra-arterial injection may lead to spinal cord infarction. Considering these complications nonparticulate steroids offers safety.

Radicular pain due to disc herniation, up to $70 \%$ of the patients achieve $50 \%$ relief of pain between 1 and 2 months, about $40 \%$ patients maintain this outcome for 12 months and about 30\% achieve complete relief. Transforaminal epidural steroid injection (TFESI) is more often successful in contained herniations or patient with low grade compressions. More effective in acute pain and marginally effective in chronic pain. It reduces the burden of disease, along with pain relief it helps in restoration of functions and reduces the need for surgery. Transforaminal epidural steroid injection is not a surgical procedure and should not be judged according to the expectations of a surgical procedure.

For chronic radicular pain DRG pulsed radiofrequency lesioning (PRFL), as a non-destructive neuromodulation technique, would be more attractive for chronic pain management. Although the results of pulsed radiofrequency lesioning (PRFL) for neuropathic pain are anecdotal, the technique can be used as a potential treatment for chronic neuropathic pain.

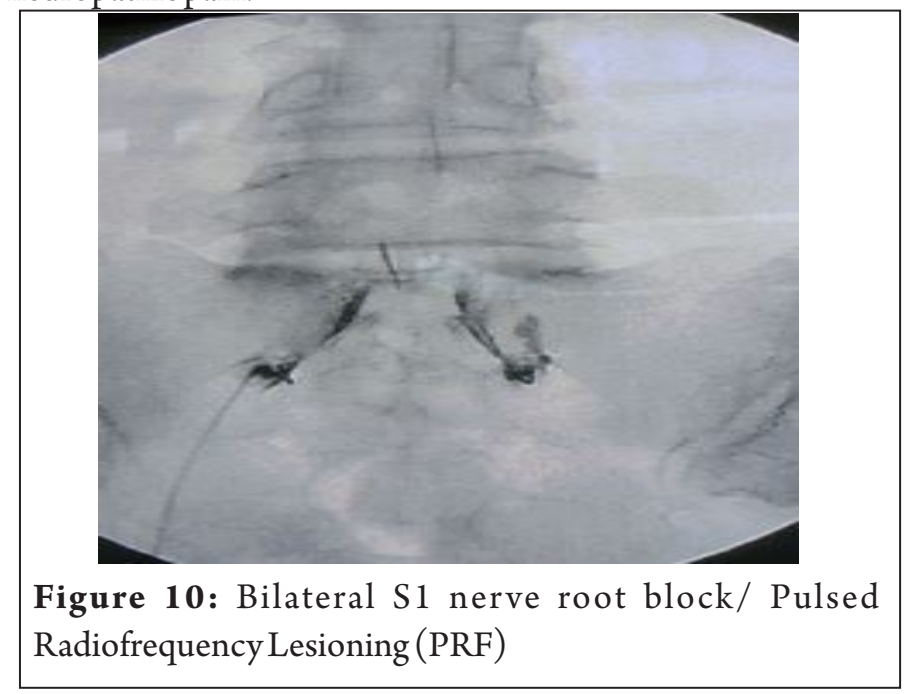

Facet Joint Injection [6] [7]

Procedure in which needle is introduced inside the cavity of facetjoint.

Articular surface of joint is straight and flat in longitudinal section where as it is flat or curved in transverse section. Flat joints are oriented at various degrees to sagittal plane (e.g. L2-3 is $15^{\circ}, \mathrm{L} 3-430^{\circ}, \mathrm{L} 4-5, \& \mathrm{~L} 5-\mathrm{S} 1$ is $45^{\circ}$ ). With flat joint while doing the oblique rotation we can achieve optimal lucency at one particular angle which is optimal for needle entry whereas for curved joint radiolucency can be seen at various angulations, out of that first image where maximal lucency is seen should be selected for needle entry otherwise entry to joint won't be possible.

Capsule of the joint is lax at superior and inferior ends which forms subcapsular recess, this is the site we can gain entry to joint if it's not possible from centre of joint.

Each facet joint is supplied by two median nerve (eg. facet joint L4-5 is supplied by median branch coming from L3 \& L4 nerve root)

\section{Technique}

Under prone position, under local anaesthesia with $\mathrm{C}$-arm guidance procedure is to be performed. Superior end plate squaring is to be adjusted first. Later on $\mathrm{C}$-arm should be tilted transversely until maximum radiolucent joint space is observed. Entry point is to be marked on skin and with tunnel vision midpoint of the joint just lateral to superior articular process is targeted with $23 \mathrm{G}$ spinal needle. Access to joint can be understood by grip of needle, "give" sensation or loss of resistance or tip appears bending. $0.2 \mathrm{ml}$ contrast should be injected which can outline joint surface along with subcapsular recess. Dye may spread to subsequent facet joint due to communication, may reach to epidural space also if it is rupture.

\section{Clinical Application}

Radiologically abnormality can be seen as reduced joint space,

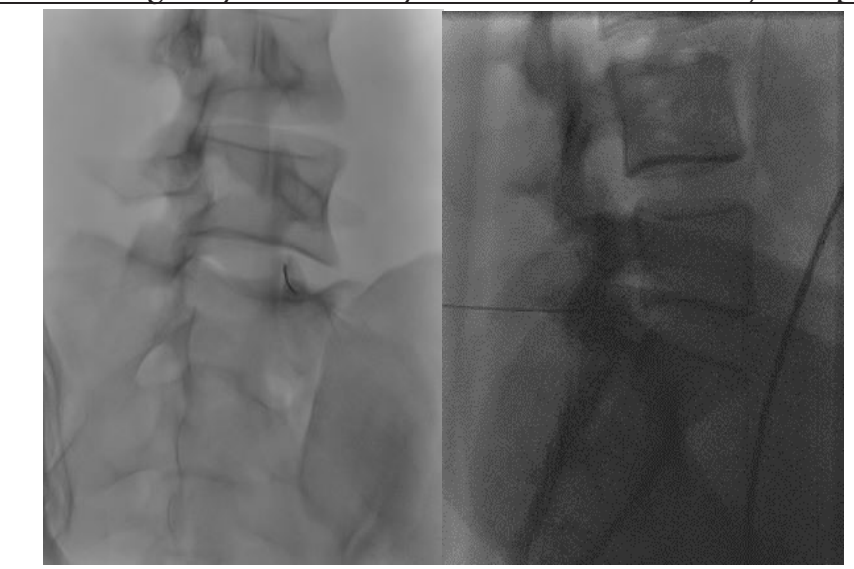

Figure 11: Right L5-S1 facet joint injection (Intra-articular) 1 Oblique view 2 Lateral view

38 | Back Bone: The Spine Journal | Volume 1 | Issue 1 | April-September 2021 | Page 33-42 
osteophytes formation, fusion, hypertrophy or subluxation. This may not be correlated with facet joint pain. So looking to the anatomical abnormality is a futile exercise. Minimal arthrography means injection is into the joint cavity. Complete arthrography need more volume tobe injected and it delineates capsule also and dye can be seen escape through defect in the capsule. It has got diagnostic value when SOL (space occupying lesion) is in intervertebral foramen whether it is communicated or not with joint. As per the current evidences and literature injection of local anesthetic to facet joint doesn't carry any diagnostic value and intra articular steroid injection is also not recommended for therapeutic effect.

Lumbar facet joint access can be used in management of synovial cyst coming from joint. Cyst can be distended and ruptured by volume and pressure or it can simply be aspirated. Steroid injections after aspiration carries therapeutic benefit.

\section{LumbarMedial Branch Block [6] [7]}

This procedure involves injection of small volume of local anesthetic to the targeted nerve/nerves to block the facet nerve supply based on hypothesis that patient's pain is coming from facet joint.

Facet joint syndrome cannot be diagnosed clinically or radiographically but can be identified with medial branch block. During examination of patient if we find that

a) Pain aggravation on hyperextension and rotation of spine and alleviation on spine flexion

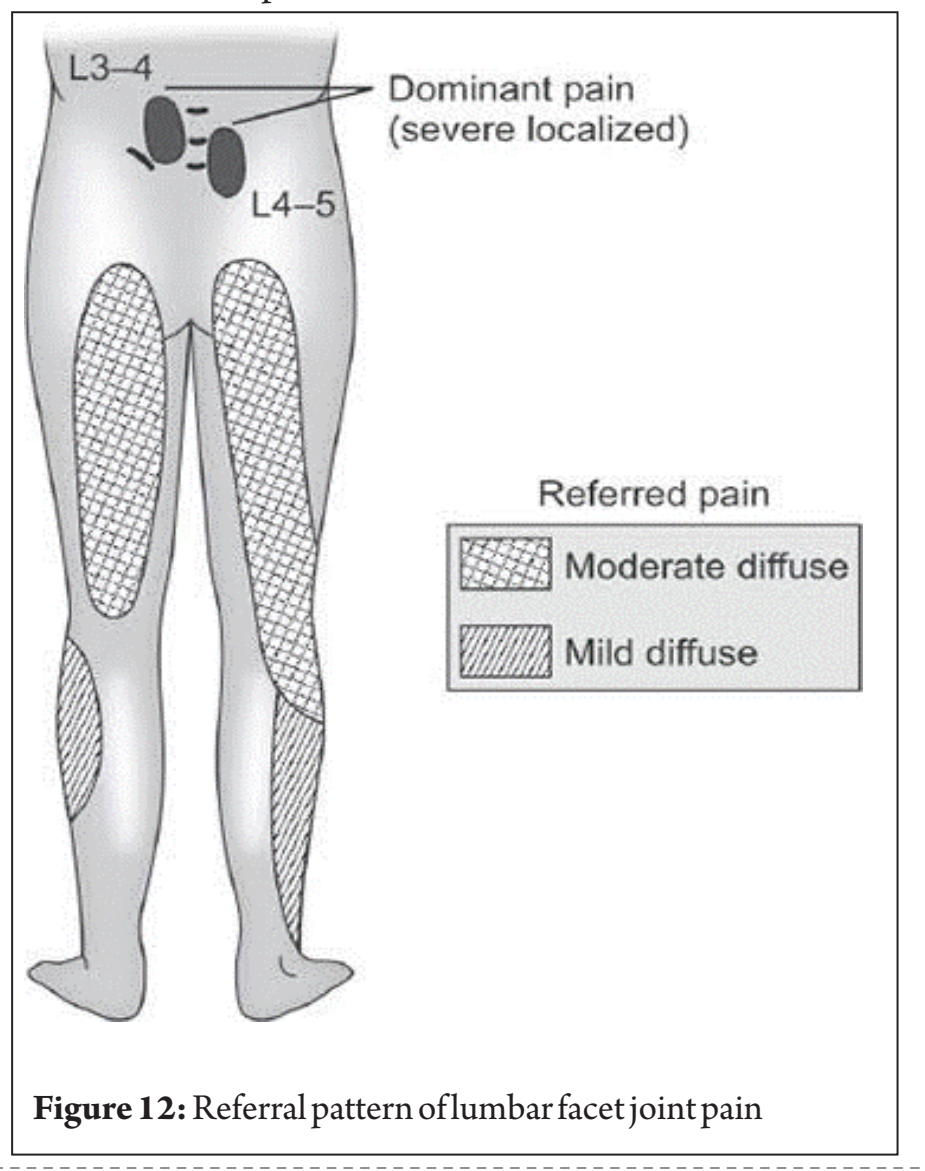

b) Local deep tenderness over the facet joint (Paraspinal)

c) Specific referral pain pattern shown in diagram

immediately decision should be made to give diagnostic median branch block to identify facet joint as a source of pain.

When radiologic signs such as severe scoliosis, osteoporosis, vertebral compression fracture, and facet osteoarthritis are present, these patients are also to be included for diagnostic block.

SPACT (single photon emission computed tomography) gives more information about facet pain compare to plain radiograph, CT or MRI.

Medial branch block is validated and it shows therapeutic value as we can proceed for radiofrequency ablation once medial branch block is positive. Injection of local anesthetic at medial branch carries only diagnostic value and should not be confused with therapeutic procedure. Positive response can be false positive in 25 to $45 \%$ of cases if only one diagnostic block is given, so ideally two diagnostic medial branch block will be required to minimise false positive response and both the time different local anesthetic drug should be chosen so pain relief should corresponds with duration of local anesthetics, it is known as concordant response where as in discordant response pain relief occurs at both the occasion but doesn't corresponds with the duration of local. We must realize that one in four responses may be false, it can be further minimise by offering three blocks, first is with local, second and third block will be like double blind placebo controlled with saline, patient should not get any relief when placebo is used.

Each of L1, L2, L3 \& L4 medial branches enters posterior compartment by passing through a notch at the junction of base of superior articular process and upper border of transverse process. Later on it crosses the lateral aspect of neck of superior articular process and finally bends medially under mamillo-accessory ligament. This ligament buries medial branch, it is large and sometimes ossified so medial branch is accessible only at the neck of superior articular process, between inflexion and mamillo-accessory ligament.

L5 dorsal ramus arches over ala of sacrum and passes in a groove formed by base of S1 superior articular process. L5 medial branch curves around caudal aspect of L5S1 facet joint. So at L5 level, L5 dorsal ramus is target point for diagnostic block rather than L5 medial branch.

\section{Technique}

Prone position, under LA, first squaring of superior end plate should be achieved by cranio-caudal adjustment of C-arm. Lateral rotation of $\mathrm{C}$-arm should be done in such a way that inflexion between superior articular process and transverse process is clearly seen. The inflexion and mamillary process should be identified. Target point is to be selected between this two limits approximately at the junction of proximal one third 


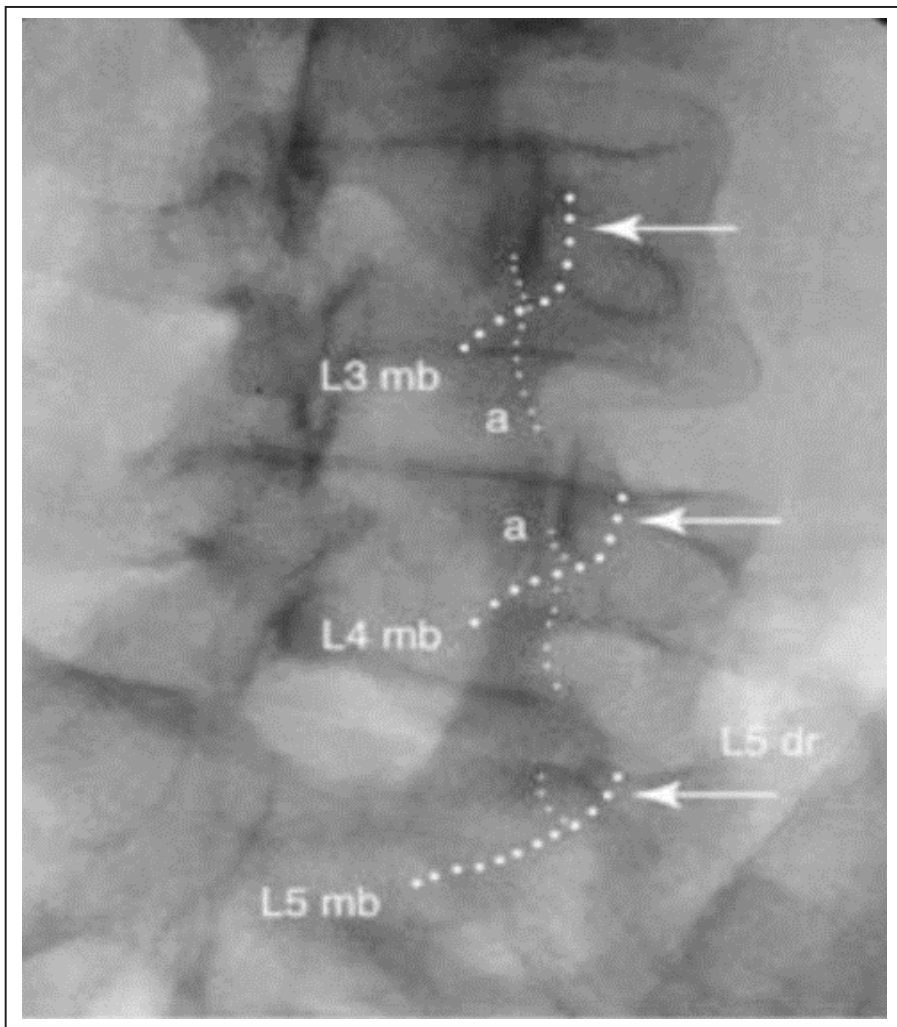

Figure 13: An oblique fluoroscopy view of the lower lumbar spine, on which have been depicted the courses of the L5 dorsal ramus (dr), the medial branches (mb) of L3, L4, and L5, and their articular branches (a). Arrows indicate the target points for medial branch blocks.

with distal two third. For L5 dorsal ramus inflexion between superior articular process of $\mathrm{S} 1$ and ala of sacrum. Adjustment of C-arm may be required as both targets can't be seen correctly on a single oblique view.

Once needle touches the bone, check the tip in PA view. The tip should be seen slightly medial to the margin of superior articular process. Declined view is obtained by declining Carm caudally in which tip of needle is seen pointing into notch between superior articular process and transverse process. Small volume ( 0.1 to $0.3 \mathrm{ml}$ ) of contrast can be injected to rule out vascular uptake and ensure adequate spread. Once satisfactory placement is achieved, $0.5 \mathrm{ml}$ LA should be injected. If relief occurs, patient should carefully attempt those movements and activities that is painful before LA injection. Relief of pain must be documented. Assessment of pain should be done at 5 to $10 \mathrm{~min}$. later and at $30 \mathrm{~min}$. later. Sometimes relief is very evident immediately. 30 minutes later if relief is not observed block should be labelled as negative. Relief of pain alone is insufficient, patient should be able to demonstrate undertaking activities which are painful before, such response is considered as in lateral view by nerves other than the ones anesthetized, there should be no relief of pain. It is preferable to diagnose multi-level pain as a staged procedure.

Same points are targeted for radiofrequency ablation but placement should be more oblique at 45 degree starting from one spacelower

\section{Radiofrequency Ablation [6] [7]}

It is percutaneous therapeutic procedure. Procedure doesn't treat actual cause of pain, it provides pain relief by anaesthetizing the source of pain. This is the reason, procedure is palliative in nature. Structures innervated by medial branch, dorsal ramus are facet joints, multifidus muscles, interspinal muscles and interspinal ligaments. There are no known cause of chronic back pain that selectively affect only individual segments of these structures, facet joints are the likely source of chronic back pain mediated by medial branches.

Radiographic anatomy should be very clear before proceeding for radiofrequency lesion. On oblique view, medial branch runs across junction between superior articular process \& transverse process. In lateral view, medial branch runs across neck of superior articular process (narrow portion of superior articular process between its head and pedicle). In this view, medial branch runs obliquely at about $45^{\circ}$ to the plane of pedicle. In declined view, medial branch appears as end on view, lying in sulcus between superior articular process and transverse process.

It is mandatory to put thermocouple electrode parallel and close to the nerve to produce sufficient lesion. In an AP view, distal portion of medial branch is covered by mamilloaccessory ligament, which is often ossified, which prevents placement of probe parallel to nerve. So electrodes must be inserted along the oblique view approximately $25^{\circ}$ from sagittal plane. In lateral view, nerve runs obliquely in caudal and dorsal direction, so electrode must be inserted along steep trajectory from below. Electrodes need to cross neck of superior articular process at $45^{\circ}$ to the transverse plane of vertebra.

\section{Technique}

General Anaesthesia is contraindicated for this procedure.

Endplate squaring of targeted segment should be done first. Followed by oblique rotation to identify target point to block

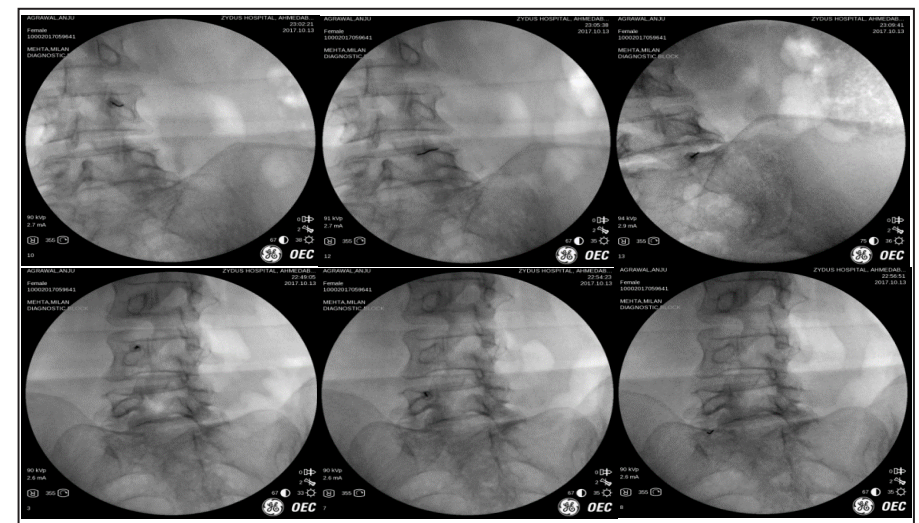

Figure 14: Median branch block at bilateral L3, L4 and Dorsal ramus of L5

40 | Back Bone: The Spine Journal | Volume 1 | Issue 1 | April-September 2021 | Page 33-42 
the medial branch. Block needle should be left in place and next step is to introduce electrode in tunnel vision. Cannula with electrode can be introduced under LA even without separate block needle and LA can be given at target point just before radiofrequency lesion. If block needle is introduced separately, it helps while introducing cannula with electrode to the targeted point as electrode is angulated approximately to $45^{\circ}$ to keep the best parallel contact with targeted nerve. C-arm should be declined approximately to $40^{\circ}$ and lateral tilt to identify sulcus. Skin entry point is to be decided. Under LA electrode is introduced and first targeted either at caudal edge of superior articular process or onto transverse process. Once bone is contacted, needle is redirected to pass into the sulcus up to few millimetres only. Depth can be confirmed in AP or oblique view. The electrode should slip along superior articular process, it should not scrape the bone of superior articular process. Finally, LA from block needle should be injected and lesion is to be done at 80 to $85^{\circ} \mathrm{C}$ for 90 seconds. Single lesion with $10 \mathrm{~mm}$ active tip electrode is sufficient. Curved tip electrode is a better choice to creat more lesions as it can be rotated clockwise and anti clock wise to create additional lesions. Post procedure patient may feel weakness in lower extremities due to spread of LA to ventral ramus. This is a very safe procedure and with the recommended technique no major complications have been reported.

Diagnostic block should offer $80 \%$ relief, based on this criteria success rate of radiofrequency procedure is very high. If single diagnostic block is used, success rate decreases drastically. If diagnostic block offers 100\% relief, success of radiofrequency by mentioned technique is very high.

\section{Sacro-iliac Joint Injection [6] [7]}

The sacroiliac joint (SIJ) is the largest axial joint in the body with an average surface area of $17.5 \mathrm{~cm}^{2}$. It is a large, auricularshaped, diarthrodial synovial joint. Only the anterior third of the interface between the sacrum and ilium is a true synovial joint; the rest of the junction is comprised of an intricate set of ligamentous connections.

If the joints become painful, they may cause pain in the low back, buttocks, abdomen, groin or legs. Typically, SIJ dysfunction is initially symptomatic after a minor traumatic event, such as a fall onto the buttocks or a slip while pushing a heavy object. It is then aggravated by transitional activities, such as climbing stairs, getting up from a chair, and getting out of a car. Pain in the SIJ can also be provoked by activities requiring asymmetrical loading through the lower extremities or pelvis, such as skating, gymnastics, golfing, and step aerobics.

The total joint volume is in range of $0.8 \mathrm{cc}$ to $2.5 \mathrm{cc}$.

Prevalence is about $20 \%$ and it is about $30 \%$ after lumbar fusion surgery.
No clinical feature is diagnostic, however if three to four provocative tests are positive, positive response to block is most likely occur.

\section{Technique}

Prone position, under LA, with all standard preparation first check AP view. In AP view decision can't be made which one is posterior margin and which one is anterior. 1 to $2 \mathrm{~cm}$ from most inferior end is our target area for an injection. First objective is to identify posterior margin. Contralateral oblique view is very useful as all margins superimposed. Joint space appears as most translucent and relatively white for that 5 to $20^{\circ}$ contra lateral adjustment is required. At times, 20 to $25^{\circ}$ cephalad tilt is required to differentiate posterior joint plane from anterior joint plane. Skin entry point is decided and needle should be guided in tunnel vision. First target towards sacrum, then withdraw a little and redirected towards joint space. Loss of resistance and "give" sensation is felt. On fluoroscopic view tip should be seen clearly in between two bones. Intraarticular access is confirmed by dye injection. Only 0.3 to $0.5 \mathrm{ml}$ contrast will be injected. If injection of contrast is not possible to inject, tip might be inside the cartilage, in that case rotate and withdraw a bit and inject again. Sometimes it is impossible to get an arthrogram due to ankylosis. One must try at most inferior aspect where both the margins are superimposed, or else just beyond the most inferior joint, into the inferior capsular recess. At last middle or superior portion of the joint should be attempted.

Dye spread in contra lateral oblique view: it outlines perimeter of joint and forms an auricular shaped blush across the joint. On ipsilateral oblique view, linear streaks between joint margin is seen. On AP views, it fills spaces between various margin. Lateral view of arthrogram is delineate joint space and appears as auricular shape of joint.

After achieving correct arthrogram LA can be injected and this procedure is only diagnostic block. Two types of control are used for SIJ blocks.

1) Anatomical control: The sacroiliac block is deemed positive if blocking the joint relieves the pain but, blocking the adjacent structure doesn't. The block is negative if blocking nearby

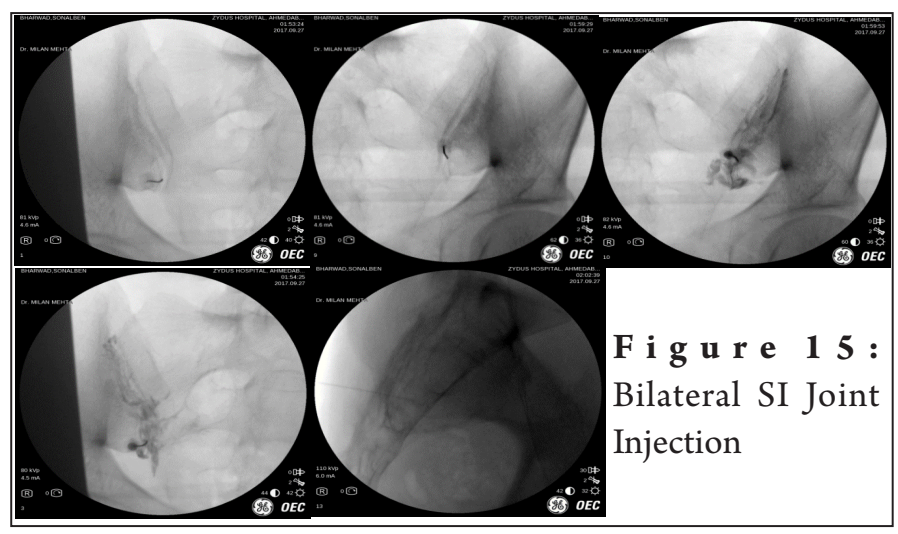

41 | Back Bone: The Spine Journal | Volume 1 | Issue 1 | April-September 2021 | Page 33-42 
structure relieves pain irrespective of the response to sacroiliac block.

2) Physiological control: block should be given at two different occasion with different LA and relief should corresponds to the duration of that particular LA. One can go for more vigorous approach by placebo controlled blocks.

Diagnostic block can be used prior to steroid injection or prior to Fusion.

Evidence for steroid injection is stronger for pain of inflammatory origin and it's weaker for idiopathic pain.

\section{References}

1. Bartleson JD, Maus TP. Diagnostic and therapeutic spinal interventions. NeurolClinPract.2014Aug; 4(4):347-352.

2. Buy X, Gangi A. Percutaneous Treatment of Intervertebral Disc Herniation. SeminInterventRadiol.2010Jun; 27(2): 148-159.

3. Diwan S, Sayed D, Deer T, Salomons A, Liang K. An Algorithmic Approach to Treating Lumbar Spinal Stenosis: An Evidenced-Based Approach. Pain Med. 2019Dec;20(Suppl2): S23-S31.

4. Schoenfeld AJ, Swiner BK. Treatment of lumbar disc herniation: Evidence-based
practice.IntJGenMed.2010; 3: 209-214.

5. Parikh KS, Seetharamaiah S. Approach to failed spinal anaesthesia for caesarean section. Indian J Anaesth. 2018 Sep; 62(9): 691-697.

6. NikolalBogduk. ISIS Practice Guidelines for Spinal Diagnostic and Treatment Procedures: 2nd Edition, International Spine Intervention Society, 2014.

7. Kim DH, Kim KH, Kim YC. Minimally Invasive Percutaneous Spinal Techniques: 1stedition, Elsevier B.V publication 2011.
Conflict of Interest: NIL Source of Support: NIL

\section{How to Cite this Article}

Mehta M, Shah BJ, Chudasama P | Common Lumbar and Sacral Interventional Pain Procedures | Back Bone: The Spine Journal | April-September 2021;2(1):33-42. 